\title{
Probing the Surface of $\mathrm{La}_{0.6} \mathrm{Sr}_{0.4} \mathrm{MnO}_{3}$ in Water
}

\section{Vapor by In Situ Photon-In / Photon-Out}

\section{Spectroscopy}

Philipp Busse $1,2,4$, Zhong Yin ${ }^{1,2, \dagger}$, Daniel Mierwaldt ${ }^{4}$, Julius Scholz ${ }^{4}$, Birte Kressdorf ${ }^{4}$, Leif Glaser $^{2}$, Piter S. Miedema ${ }^{2}$, André Rothkirch ${ }^{2}$, Jens Viefhaus ${ }^{2, \dagger \dagger}$, Christian Jooss ${ }^{4,5, *}$, Simone Techert ${ }^{1,2,3,{ }^{*}, \text { and Marcel Risch }}{ }^{4,6, *}$

${ }^{1}$ Structural Dynamics of (Bio)chemical Systems, Max-Planck-Institute for Biophysical Chemistry Göttingen, 37077 Germany

${ }^{2}$ Photon Science, Deutsches Elektronen-Synchrotron DESY, Hamburg, 22607 Germany ${ }^{3}$ Institute of X-ray Physics, University of Göttingen, 37077 Germany ${ }^{4}$ Institute of Materials Physics, University of Göttingen, 37077 Germany

${ }^{5}$ International Center for Advanced Energy Studies, University of Göttingen, Tammannstr. 4, 37077 Göttingen, Germany

${ }^{6}$ Helmholtz-Zentrum Berlin für Materialien und Energie GmbH, Nachwuchsgruppe Gestaltung des Sauerstoffentwicklungsmechanismus, 14109 Berlin, Germany

\section{Corresponding authors}

* Marcel Risch, marcel.risch@helmholtz-berlin.de

* Simone Techert, simone.techert@desy.de

*Christian Jooss, cjooss@gwdg.de

10 pages, 7 supporting figures, 4 supporting tables 


\section{Supporting Figures}
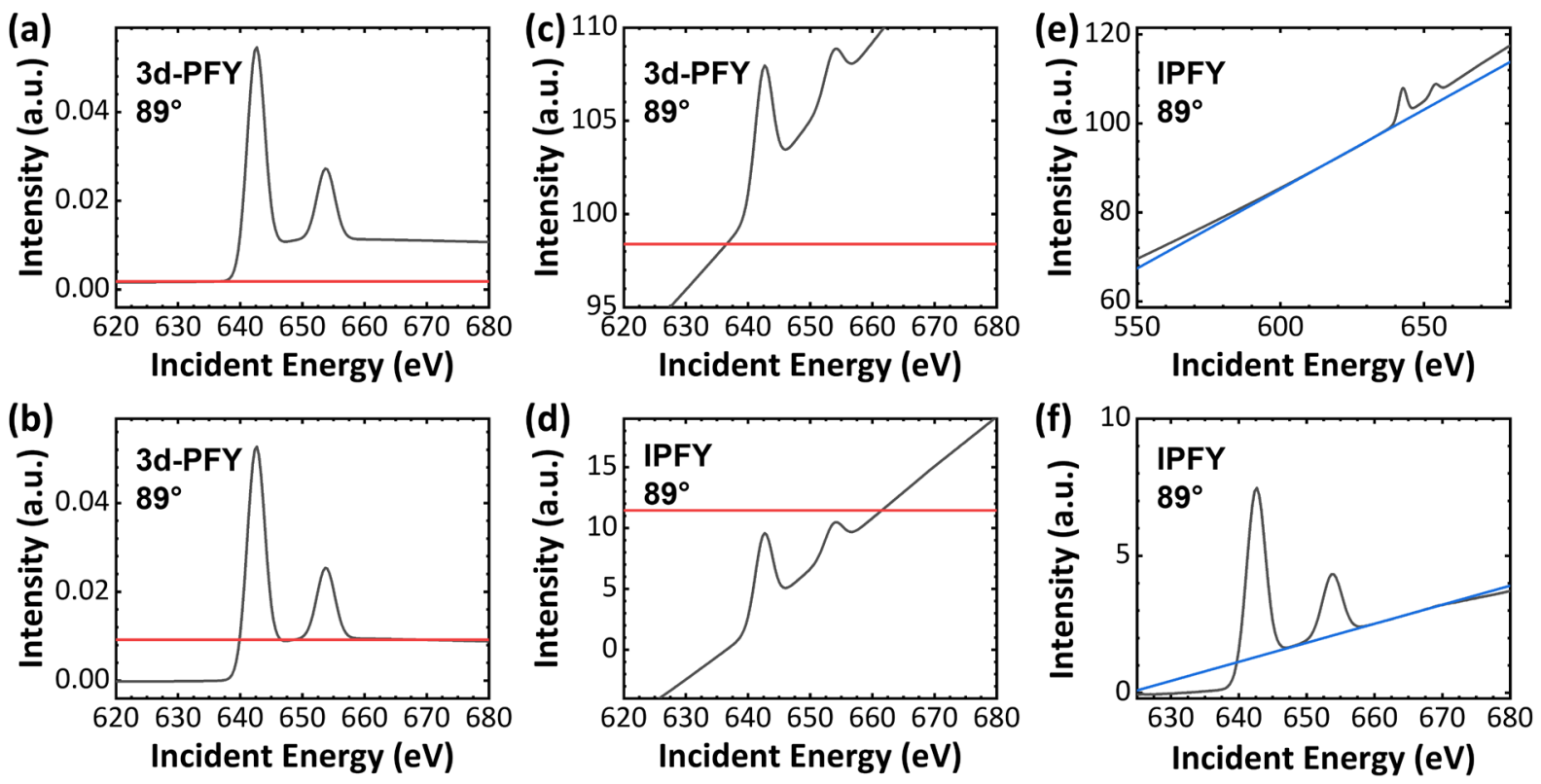

Figure S1. Illustration of the normalization procedure. (a) Subtraction of the average intensity between 634 and $638 \mathrm{eV}$, followed by (b) division of the average intensity between 658 and $670 \mathrm{eV}$ for the $3 \mathrm{~d}-\mathrm{PFY}$ simulation at $89^{\circ}$ incident angle. (c) Subtraction of the average intensity between 635 and $638 \mathrm{eV}$, followed by (d) division of the average intensity between 658 and $665 \mathrm{eV}$ for the IPFY simulation at $89^{\circ}$ incident angle. The normalization function is clearly unsuitable for the IPFY simulations. Alternative normalization procedure of (e) Subtraction of a linear function $(0.36 \mathrm{E}-129 \mathrm{eV})$ matching the intensity between 609.00 and $637.33 \mathrm{eV}$, followed by (f) division of a linear function $(0.07 \mathrm{E}-43 \mathrm{eV})$ matching the intensity between 659.00 and $667.67 \mathrm{eV}$. The final normalized spectra may be found in Fig. 3 of the main text. Details and parameters of the simulations may be found in Table S1.

When linear functions are used in the normalization, the normalized peak intensities are much less reduced as compared to $\mu_{M n}$ and the $\mathrm{L}_{3} / \mathrm{L}_{2}$ ratio is 2.73 at $89^{\circ}$. Yet, deviations at energies lower and higher than the fit window suggest that a higher order polynomial may give better results but it would require to record a larger energy window. The amplitudes of the IPFY were also lowest among the experimental PFYs as discussed below. Thus, we suggest to record lower excitation energies before the $\mathrm{L}_{3}$ edge as commonly reported in order to fit a suitable polynomial for the correct normalization of the IPFY data. 

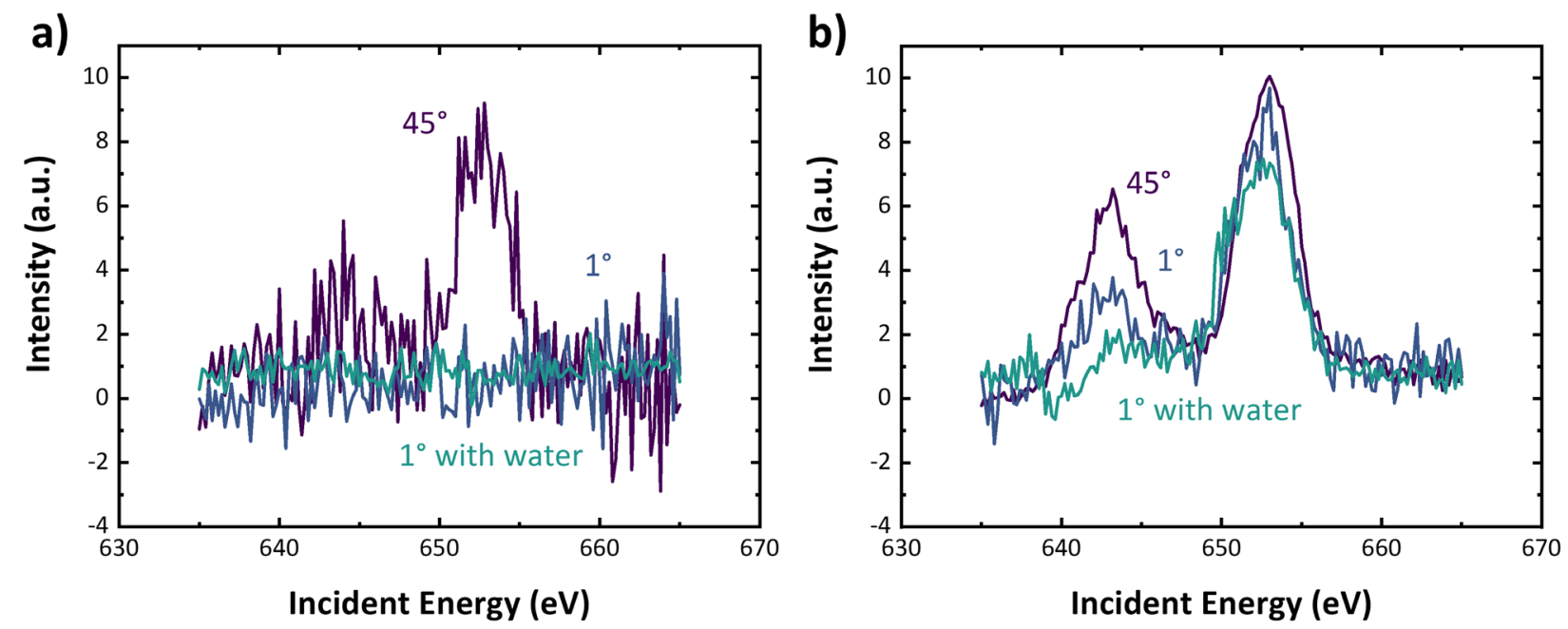

Figure S2. Comparison of (a) the VLS-TFY (integration over all emission energy of the VLS grating) with (b) the $\Sigma$-PFY (sum of only the Mn3d-PFY, Mn3s-PFY and O-PFY ROIs). The VLS-TFY shows intensities for $\alpha=45^{\circ}$ (violet) only. For the surface-sensitive geometry (blue and green) the total fluorescence of manganese is compensated by the inverse signal of oxygen to annihilation of the VLS-TFY signal. The color codes are equal for (a) and (b).
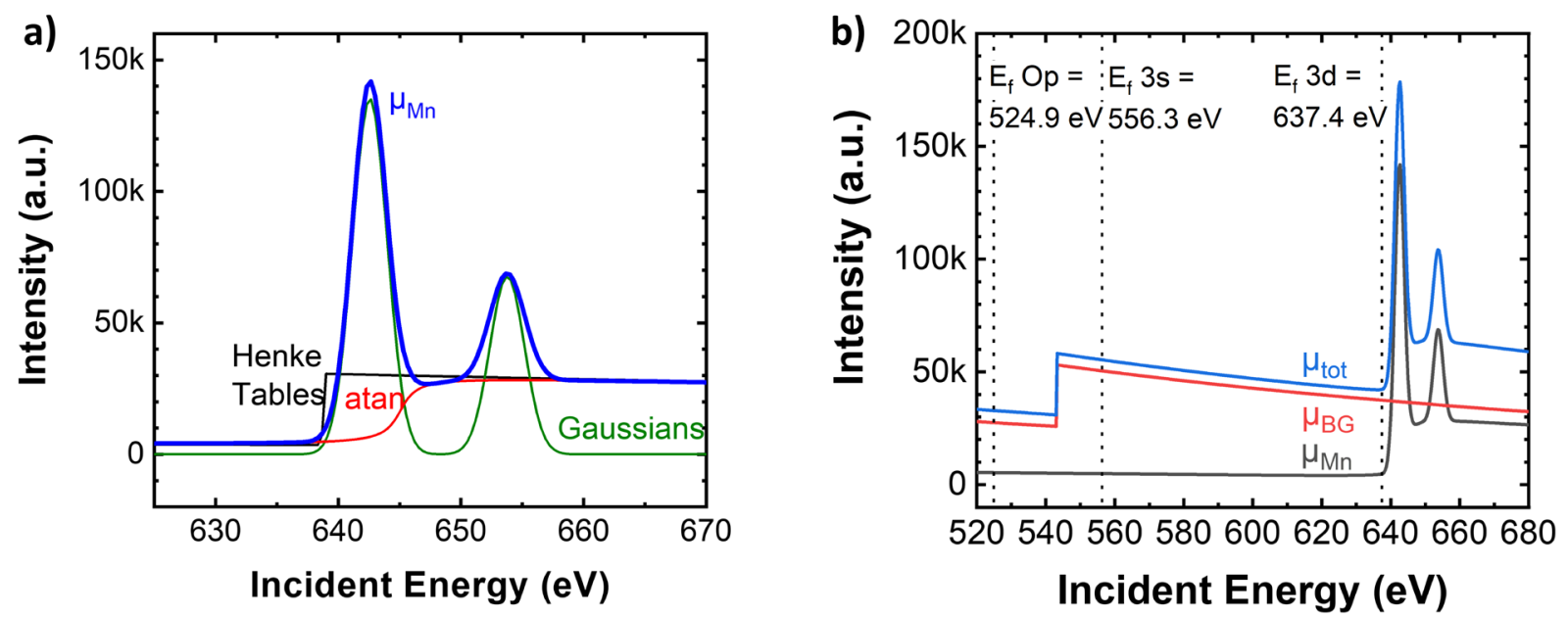

Figure S3. (a) Construction of $\mu_{\mathrm{Mn}}$ from the Henke Tables ${ }^{1}$ and an atan function to simulate the edge jump and Gaussians to approximate the multiplet structure. (b) Construction of $\mu_{\text {tot }}$ from $\mu_{\mathrm{Mn}}$ as shown on the left and $\mu_{\mathrm{bg}}=\mu_{\mathrm{O}}+\mu_{\mathrm{La}}+\mu_{\mathrm{Sr}}$ from the Henke Tables ${ }^{1}$. 


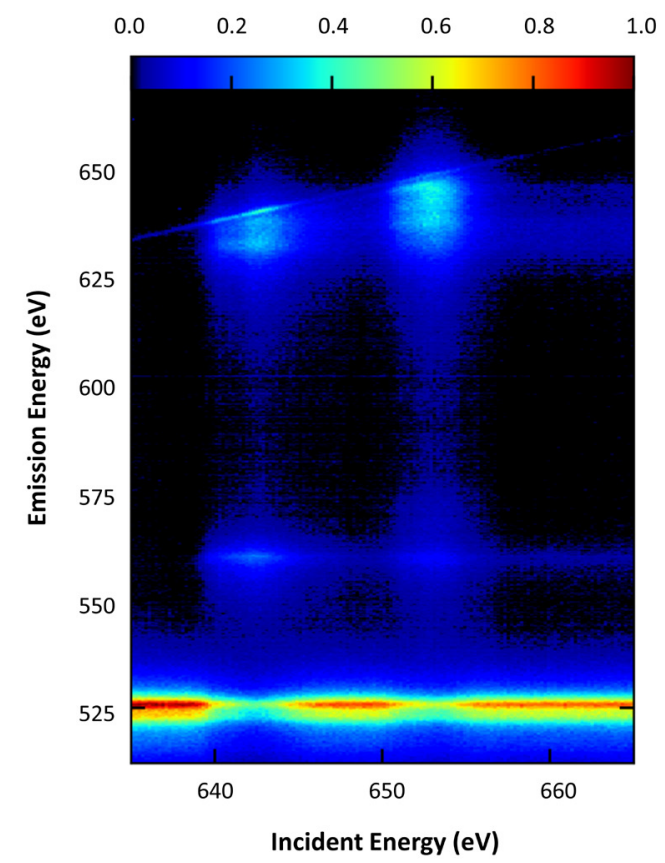

Figure S4. As-measured data of the RIXS-map recorded at $\alpha=45^{\circ}$ corresponding to the smoothed RIXS map shown in Fig. 4 of the main text.
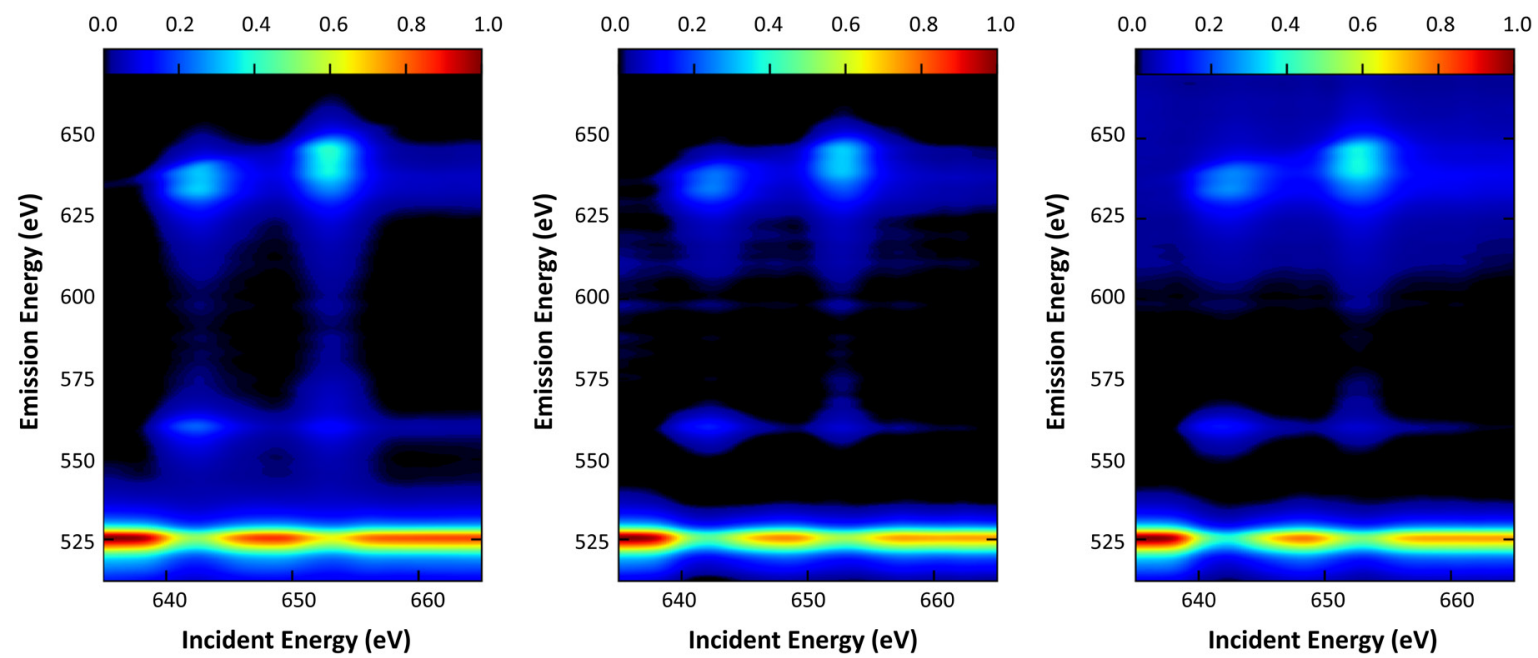

Figure S5. The RIXS-maps used for the extraction of all the experimental PFY spectra from the main text recorded at (a) $45^{\circ}$, (b) $1^{\circ}$ and (c) $1^{\circ}$ with additional water. The data shown here was smoothed as in Fig. 4 of the main text for clarity. The scales shown here are all relative (minimum $=0$, maximum $=1$ ). The absolute intensities differed. The elastic line was used for the callibration of the emission energy axis. 
a)

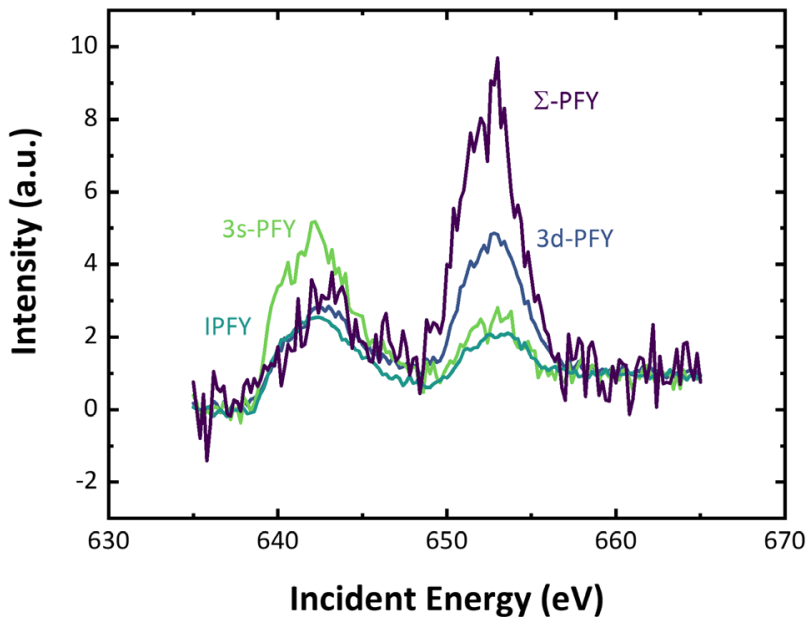

b)

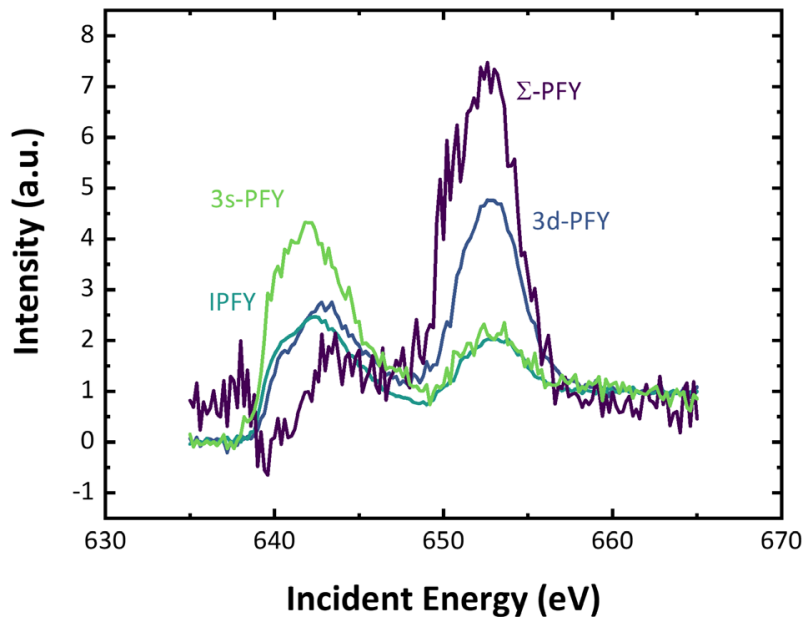

Figure S6. (a) The spectra of the different PFYs for $\alpha=1^{\circ}$ with only a little amount of water (low vacuum condition) and (b) for $\alpha=1^{\circ}$ with further water added to the surface (in-situ conditions). 

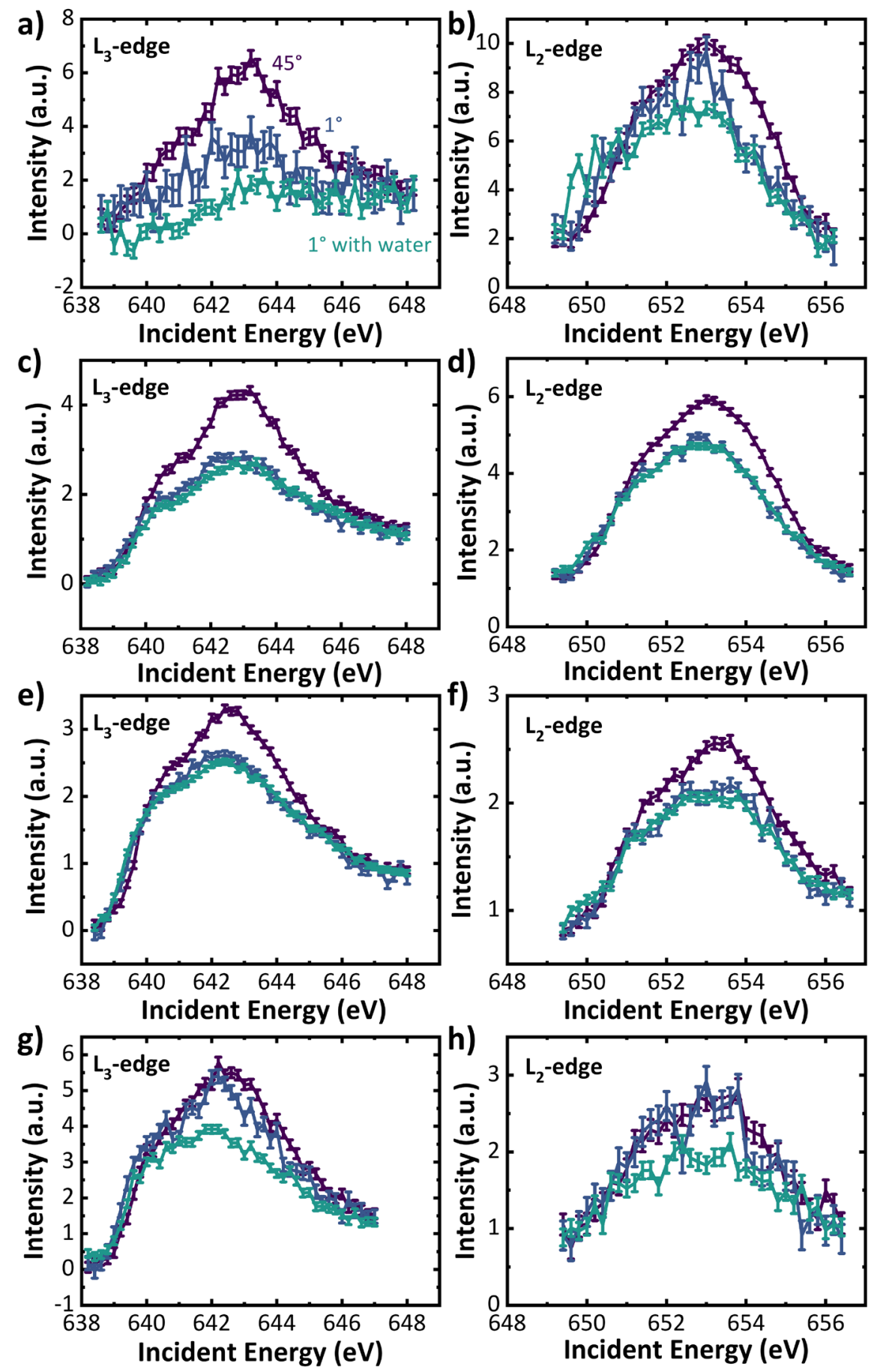

Figure S7. Comparison of changes at the L-edges with inserted noise levels (spread of multiple recorded spectra) for $\left(1^{\text {st }}\right.$ row $)$ the $\Sigma$-PFY, $\left(2^{\text {nd }}\right.$ row $)$ the $3 \mathrm{~d}-\mathrm{PFY},\left(3^{\text {rd }}\right.$ row $)$ the IPFY and $\left(4^{\text {th }}\right.$ row) the 3s-PFY. The color codes are equal for all subfigures. The relatively large error bars for the 3s-PFY under grazing incidence with only a little amount of water at the surface (blue) are a result of a drifting beam during the experiment. 


\section{Supporting Tables}

Table S1. Parameters and formulas used to produce the simulations in Fig. 3 of the main text.

\begin{tabular}{|c|c|c|}
\hline & \multicolumn{2}{|l|}{ Formula } \\
\hline atan'(E) & \multicolumn{2}{|c|}{$\begin{array}{l}\mathrm{d} \mu / \mathrm{d} E_{\text {post }}(E)\left(\operatorname{atan}\left(E-E_{L 3}-E_{\text {edge }}\right) / \pi+1 / 2\right)+ \\
\mathrm{d} \mu / \mathrm{dE}_{\text {pre }}(\mathrm{E})\left(1-\operatorname{atan}\left(E-E_{L 3}-E_{\text {edge }}\right) / \pi+1 / 2\right)\end{array}$} \\
\hline$\mu_{M n}(E)$ & \multicolumn{2}{|c|}{$\begin{array}{l}\operatorname{atan}^{\prime}(\mathrm{E})+w_{\mu} \Delta \mu_{\mathrm{Mn}} \exp \left(-\left(\mathrm{E}-E_{f}-E_{\text {edge }}-E_{\text {offset }}\right)^{2} / 2 / \sigma^{2}\right)+ \\
\left(\mathrm{w}_{\mu} / \mathrm{r}_{\mathrm{L} 3 \mathrm{~L} 2}-1\right) \Delta \mu \exp \left(-\left(\mathrm{E}-E_{f}-E_{\text {edge }}-E_{\text {offset }}\right)^{2} / 2 / \sigma^{2}\right)\end{array}$} \\
\hline$\mu_{t o t}(E)$ & \multicolumn{2}{|c|}{$\mu_{M n}(\mathrm{E})+w_{b g}\left(\mu_{O}(\mathrm{E})+\mu_{L a}(\mathrm{E})+\mu_{S r}(\mathrm{E})\right)$} \\
\hline $3 \mathrm{~d}-\mathrm{PFY} *$ & \multicolumn{2}{|c|}{$\begin{array}{l}\left.\left.\mu_{M n}(E) / \mu_{t o t}(E)+\mu_{t o t}\left(E_{f, 3 d}\right)\left(\sin (\alpha) / \sin \left(90^{\circ}-\alpha\right)\right)\right)\right) \\
\left(1-\exp \left(-d\left(\mu_{t o t}(E) / \sin (\sin (\alpha))+\mu_{t o t}\left(E_{f, 3 d}\right) / \sin \left(90^{\circ}-\alpha\right)\right)\right)\right)\end{array}$} \\
\hline $3 \mathrm{~s}-\mathrm{PFY} *$ & \multicolumn{2}{|l|}{$\mu_{M n}(E) / \sin (\alpha)$} \\
\hline IPFY* & \multicolumn{2}{|c|}{$\begin{array}{l}\left.1 /\left(\mu_{O}(E) / \mu_{t o t}(E)+\mu\left(E_{f, O}\right)\left(\sin (\alpha) / \sin \left(90^{\circ}-\alpha\right)\right)\right)\right) \\
\left.\left(1-\exp \left(-d\left(\mu_{t o t}(E) / \sin (\sin (\alpha))+\mu\left(E_{f, O}\right) / \sin \left(90^{\circ}-\alpha\right)\right)\right)\right)\right)\end{array}$} \\
\hline Parameter & Value & Comment \\
\hline $\mathrm{d} \mu / \mathrm{d} E_{\text {post }}(E)$ & Fig. S2 & $\begin{array}{l}\text { Slope of } \mu_{\mathrm{Mn}} \text { from Henke tables }{ }^{1} \text { after the } \\
\mathrm{L}_{3} \text { edge }\end{array}$ \\
\hline $\mathrm{d} \mu / \mathrm{d} E_{p r e}(E)$ & Fig. S2 & $\begin{array}{l}\text { Slope of } \mu_{\mathrm{Mn}} \text { from Henke tables }{ }^{1} \text { before } \\
\text { the } \mathrm{L}_{3} \text { edge }\end{array}$ \\
\hline$E_{L 3}$ & $638.7 \mathrm{eV}$ & Edge position of Mn (metal) \\
\hline$E_{\text {edge }}$ & $2.5 \mathrm{eV}$ & Estimated edge shift of the oxide \\
\hline$w_{\mu}$ & 5.0 & Scaling factor for Gaussian peaks \\
\hline $\mathrm{r}_{\mathrm{L} 3 \mathrm{~L} 2}$ & 2.0 & Ratio of $\mathrm{L}_{3}$ to $\mathrm{L}_{2}$ height \\
\hline$\Delta \mu_{\mathrm{Mn}}$ & 27069.68 & Edge step in $\mu_{M n}$ from Henke Tables ${ }^{1}$ \\
\hline$E_{\text {offset }}$ & $3.87 \mathrm{eV}$ & Shift to coarsely match experimental data \\
\hline$\sigma^{2}$ & $2 \mathrm{eV}^{2}$ & Width parameter of Gaussian \\
\hline$\mu_{b g}$ & Fig. 3 and Table S2 & Weight of background contribution \\
\hline$\mu_{O}(\mathrm{E}), \mu_{L a}(\mathrm{E}), \mu_{S r}(\mathrm{E})$ & Fig. S2 & Absorption from Henke Tables ${ }^{1}$ \\
\hline$\mu_{\text {tot }}\left(E_{f, 3 d}\right)$ & 42247.880; Fig. S3 & $\begin{array}{l}\text { Absorption at fluorescence energy of the } \\
\text { Mn 3d-2p transition }\end{array}$ \\
\hline$\mu_{t o t}\left(E_{f, O}\right)$ & 32862.079; Fig. S3 & $\begin{array}{l}\text { Absorption at fluorescence energy of the } \\
\text { O } 2 p-1 s \text { transition }\end{array}$ \\
\hline$\alpha$ & Fig. 3 and Table S2 & Incident angle \\
\hline$d$ & $80 \mathrm{~nm}$ & Sample thickness \\
\hline
\end{tabular}

* The normalization procedure is detailed in the experimental section of the main text and illustrated in Fig. S2. 
Table S2. L3/L2 ratios of the simulations in Fig. 3 of the main text.

\begin{tabular}{|c|c|c|c|c|c|c|c|}
\hline & $\begin{array}{l}\mathrm{L}_{3} \text { height at } \\
642.57 \mathrm{eV}\end{array}$ & $\begin{array}{l}L_{2} \text { height at } \\
653.67 \mathrm{eV}\end{array}$ & $\begin{array}{l}\mathrm{L}_{3} / \mathrm{L}_{2} \\
\text { ratio }\end{array}$ & & $\begin{array}{l}L_{3} \text { height at } \\
642.57 \mathrm{eV}\end{array}$ & $\begin{array}{l}L_{2} \text { height at } \\
653.67 \mathrm{eV}\end{array}$ & $\begin{array}{l}\mathrm{L}_{3} / \mathrm{L}_{2} \\
\text { ratio }\end{array}$ \\
\hline$\mu_{M n}$ & 5.897 & 2.759 & 2.14 & $\mu_{M n}$ & 5.897 & 2.759 & 2.14 \\
\hline $3 d-P F Y$ & & & & $3 d-P F Y$ & & & \\
\hline $89^{\circ}, 1 \mu_{\mathrm{bg}}$ & 5.631 & 2.712 & 2.08 & $1^{\circ}, 10 \mu_{\mathrm{bg}}$ & 4.203 & 2.378 & 1.77 \\
\hline $45^{\circ}, 1 \mu_{\mathrm{bg}}$ & 3.666 & 2.287 & 1.60 & $1^{\circ}, 5 \mu_{\mathrm{bg}}$ & 2.598 & 1.879 & 1.38 \\
\hline $30^{\circ}, 1 \mu_{\mathrm{bg}}$ & 3.190 & 2.147 & 1.49 & $1^{\circ}, 2 \mu_{\mathrm{bg}}$ & 2.028 & 1.627 & 1.25 \\
\hline $20^{\circ}, 1 \mu_{\mathrm{bg}}$ & 2.744 & 1.988 & 1.38 & $1^{\circ}, 1 \mu_{\mathrm{bg}}$ & 1.645 & 1.425 & 1.15 \\
\hline $10^{\circ}, 1 \mu_{\mathrm{bg}}$ & 2.250 & 1.755 & 1.28 & $1^{\circ}, 0.1 \mu_{\mathrm{bg}}$ & 1.265 & 1.190 & 1.06 \\
\hline $1^{\circ}, 1 \mu_{\mathrm{bg}}$ & 2.028 & 1.627 & 1.25 & & & & \\
\hline $3 s-P F Y$ & & & & $3 s-P F Y$ & & & \\
\hline $89^{\circ}, 1 \mu_{\mathrm{bg}}$ & 5.897 & 2.759 & 2.14 & $1^{\circ}, 10 \mu_{\mathrm{bg}}$ & 5.897 & 2.759 & 2.14 \\
\hline $45^{\circ}, 1 \mu_{\mathrm{bg}}$ & 5.897 & 2.759 & 2.14 & $1^{\circ}, 5 \mu_{\mathrm{bg}}$ & 5.897 & 2.759 & 2.14 \\
\hline $30^{\circ}, 1 \mu_{\mathrm{bg}}$ & 5.897 & 2.759 & 2.14 & $1^{\circ}, 2 \mu_{\mathrm{bg}}$ & 5.897 & 2.759 & 2.14 \\
\hline $20^{\circ}, 1 \mu_{\mathrm{bg}}$ & 5.897 & 2.759 & 2.14 & $1^{\circ}, 1 \mu_{\mathrm{bg}}$ & 5.897 & 2.759 & 2.14 \\
\hline $10^{\circ}, 1 \mu_{\mathrm{bg}}$ & 5.897 & 2.759 & 2.14 & $1^{\circ}, 0.1 \mu_{\mathrm{bg}}$ & 5.897 & 2.759 & 2.14 \\
\hline $1^{\circ}, 1 \mu_{\mathrm{bg}}$ & 5.897 & 2.759 & 2.14 & & & & \\
\hline IPFY & & & & IPFY & & & \\
\hline $89^{\circ}, 1 \mu_{\mathrm{bg}}$ & 0.836 & 0.904 & 0.92 & $1^{\circ}, 10 \mu_{\mathrm{bg}}$ & 4.562 & 2.324 & 1.96 \\
\hline $45^{\circ}, 1 \mu_{\mathrm{bg}}$ & 3.776 & 1.938 & 1.95 & $1^{\circ}, 5 \mu_{\mathrm{bg}}$ & 5.159 & 2.551 & 2.02 \\
\hline $30^{\circ}, 1 \mu_{\mathrm{bg}}$ & 4.443 & 2.163 & 2.05 & $1^{\circ}, 2 \mu_{\mathrm{bg}}$ & 5.246 & 2.584 & 2.03 \\
\hline $20^{\circ}, 1 \mu_{\mathrm{bg}}$ & 5.028 & 2.377 & 2.12 & $1^{\circ}, 1 \mu_{\mathrm{bg}}$ & 5.294 & 2.602 & 2.03 \\
\hline $10^{\circ}, 1 \mu_{\mathrm{bg}}$ & 5.428 & 2.595 & 2.09 & $1^{\circ}, 0.1 \mu_{\mathrm{bg}}$ & 5.375 & 2.633 & 2.04 \\
\hline $1^{\circ}, 1 \mu_{\mathrm{bg}}$ & 5.246 & 2.584 & 2.03 & & & & \\
\hline $89^{\circ}, 1 \mu_{\mathrm{bg}} *$ & 5.679 & 2.081 & 2.73 & & & & \\
\hline
\end{tabular}

* normalization by linear function $(\mathrm{y}=\mathrm{ax}+\mathrm{b})$ instead of constant (as all other simulations and experimental data). 
Table S3. Parameters used to produce the models in Fig. 6 of the main text. The values were determined "by eye" to match the experimental spectrum recorded at $\alpha=45^{\circ}$.

\begin{tabular}{|c|c|c|}
\hline & \multicolumn{2}{|l|}{ Formula } \\
\hline $\operatorname{atan}(\mathrm{E})$ & \multicolumn{2}{|c|}{$\begin{array}{l}\mathrm{d} \mu / \mathrm{d} E_{\text {post }}(E)\left(\operatorname{atan}\left(E-E_{L 3}-E_{\text {edge }}\right) / \pi+1 / 2\right)+ \\
\mathrm{d} \mu / \mathrm{d} E_{\text {pre }}(\mathrm{E})\left(1-\operatorname{atan}\left(E-E_{L 3}-E_{\text {edge }}\right) / \pi+1 / 2\right)\end{array}$} \\
\hline$\mu_{M n}(E)$ & \multicolumn{2}{|c|}{$\begin{array}{l}\operatorname{atan}^{6}(\mathrm{E})+w_{\mu} \Delta \mu_{\mathrm{Mn}} \exp \left(-\left(\mathrm{E}-E_{f}-E_{\text {edge }}-E_{\text {offset }}\right)^{2} / 2 / \sigma^{2}\right)+ \\
\left(\mathrm{w}_{\mu} / \mathrm{r}_{\mathrm{L} \text { LL2 }}-1\right) \Delta \mu \exp \left(-\left(\mathrm{E}-E_{f}-E_{\text {edge }}-E_{\text {offset }}\right)^{2} / 2 / \sigma^{2}\right)\end{array}$} \\
\hline$\mu_{\text {tot }}(E)$ & \multicolumn{2}{|c|}{$\mu_{M n}(\mathrm{E})+w_{b g}\left(\mu_{O}(\mathrm{E})+\mu_{L a}(\mathrm{E})+\mu_{S r}(\mathrm{E})\right)$} \\
\hline 3d-PFY* & \multicolumn{2}{|c|}{$\begin{array}{l}\left.\left.\mu_{M n}(E) / \mu_{t o t}(E)+\mu_{t o t}\left(E_{f, 3 d}\right)\left(\sin (\alpha) / \sin \left(90^{\circ}-\alpha\right)\right)\right)\right) \\
\left(1-\exp \left(-d\left(\mu_{t o t}(E) / \sin (\sin (\alpha))+\mu_{t o t}\left(E_{f, 3 d}\right) / \sin \left(90^{\circ}-\alpha\right)\right)\right)\right)\end{array}$} \\
\hline $3 \mathrm{~s}-\mathrm{PFY} *$ & \multicolumn{2}{|l|}{$\mu_{M n}(E) / \sin (\alpha)$} \\
\hline IPFY* & \multicolumn{2}{|c|}{$\begin{array}{l}\left.1 /\left(\mu_{O}(E) / \mu_{t o t}(E)+\mu\left(E_{f, O}\right)\left(\sin (\alpha) / \sin \left(90^{\circ}-\alpha\right)\right)\right)\right) \\
\left.\left(1-\exp \left(-\mathrm{d}\left(\mu_{t o t}(E) / \sin (\sin (\alpha))+\mu\left(E_{f, O}\right) / \sin \left(90^{\circ}-\alpha\right)\right)\right)\right)\right)\end{array}$} \\
\hline Parameter & Value & Comment \\
\hline $\mathrm{d} \mu / \mathrm{d} E_{p o s t}(E)$ & Fig. S3 & $\begin{array}{l}\text { Slope of } \mu_{\mathrm{Mn}} \text { from Henke tables }{ }^{1} \text { after the } \\
\mathrm{L}_{3} \text { edge }\end{array}$ \\
\hline $\mathrm{d} \mu / \mathrm{d} E_{p r e}(E)$ & Fig. S3 & $\begin{array}{l}\text { Slope of } \mu_{\mathrm{Mn}} \text { from Henke tables }{ }^{1} \text { before } \\
\text { the } \mathrm{L}_{3} \text { edge }\end{array}$ \\
\hline$E_{L 3}$ & $638.7 \mathrm{eV}$ & Edge position of Mn (metal) \\
\hline$E_{\text {edge }}$ & $2.5 \mathrm{eV}$ & Estimated edge shift of the oxide \\
\hline$w_{\mu}$ & $\begin{array}{l}\text { 3d-PFY: } 6.6 \\
\text { 3s-PFY: } 4.4 \\
\text { IPFY: } 4.5\end{array}$ & Scaling factor for Gaussian peaks \\
\hline $\mathrm{r}_{\mathrm{L} 3 \mathrm{~L} 2}$ & $\begin{array}{l}\text { 3d-PFY: } 0.25 \\
\text { 3s-PFY: } 1.90 \\
\text { IPFY: } 1.30\end{array}$ & Ratio of $\mathrm{L}_{3}$ to $\mathrm{L}_{2}$ height \\
\hline$\Delta \mu_{\mathrm{Mn}}$ & 27069.68 & Edge step in $\mu_{M n}$ from Henke Tables ${ }^{1}$ \\
\hline$E_{\text {offset }}$ & $3.87 \mathrm{eV}$ & Shift to coarsely match experimental data \\
\hline$\sigma^{2}$ & $\begin{array}{l}\text { 3d-PFY: } 1.2 \mathrm{eV}^{2} \\
\text { 3s-PFY: } 2.0 \mathrm{eV}^{2} \\
\text { IPFY: } 2.0 \mathrm{eV}^{2}\end{array}$ & Width parameter of Gaussian \\
\hline$w_{b g}$ & 1.0 & Weight of background contribution \\
\hline$\mu_{O}(\mathrm{E}), \mu_{L a}(\mathrm{E}), \mu_{S r}(\mathrm{E})$ & Fig. S3 & Absorption from Henke Tables ${ }^{1}$ \\
\hline$\mu_{t o t}\left(E_{f, 3 d}\right)$ & 42247.880; Fig. S3 & $\begin{array}{l}\text { Absorption at fluorescence energy of the } \\
\text { Mn 3d-2p transition }\end{array}$ \\
\hline$\mu_{t o t}\left(E_{f, o}\right)$ & 32862.079; Fig. S3 & $\begin{array}{l}\text { Absorption at fluorescence energy of the } \\
\text { O 2p-1s transition }\end{array}$ \\
\hline$\alpha$ & $45^{\circ}, 1^{\circ}$ & Incident angle \\
\hline$d$ & $80 \mathrm{~nm}$ & Sample thickness \\
\hline$\Delta E_{L 3}$ & $\begin{array}{l}\text { 3d-PFY: } 0.2 \mathrm{eV} \\
\text { 3s-PFY: }-0.2 \mathrm{eV} \\
\text { IPFY: } 0.0 \mathrm{eV}\end{array}$ & Fine shift of $\mathrm{L}_{3}$ edge position \\
\hline$\Delta E_{L 2}$ & $\begin{array}{l}\text { 3d-PFY: }-0.5 \mathrm{eV} \\
\text { 3s-PFY: }-0.8 \mathrm{eV} \\
\text { IPFY: }-0.5 \mathrm{eV}\end{array}$ & Fine shift of $\mathrm{L}_{2}$ edge position \\
\hline
\end{tabular}

* The normalization procedure is detailed in the experimental section of the main text and illustrated in Fig. S2. 
Table S4. $\mathrm{L}_{3} / \mathrm{L}_{2}$ ratios of the models in Fig. 6 of the main text.

\begin{tabular}{|c|c|c|c|c|c|c|c|}
\hline Model & $\begin{array}{l}\mathrm{L}_{3} \text { height at } \\
642.57 \mathrm{eV}\end{array}$ & $\begin{array}{l}L_{2} \text { height at } \\
653.67 \mathrm{eV}\end{array}$ & $\begin{array}{l}\mathrm{L}_{3} / \mathrm{L}_{2} \\
\text { ratio }\end{array}$ & Exp & $\begin{array}{l}\mathrm{L}_{3} \text { height at } \\
642.6 \mathrm{eV}\end{array}$ & $\begin{array}{l}L_{2} \text { height at } \\
653.2 \mathrm{eV}\end{array}$ & $\begin{array}{l}\mathrm{L}_{3} / \mathrm{L}_{2} \\
\text { ratio }\end{array}$ \\
\hline $3 d-P F Y$ & & & & $3 d-P F Y$ & & & \\
\hline$\mu_{M n}$ & 7.722 & 28.579 & 0.27 & & & & \\
\hline $45^{\circ}, 1 \mu_{\mathrm{bg}}$ & 4.155 & 5.674 & 0.73 & $45^{\circ}$ & 4.183 & 5.827 & 0.72 \\
\hline $1^{\circ}, 1 \mu_{\mathrm{bg}}$ & 2.142 & 2.489 & 0.86 & $1^{\circ}$ & 2.810 & 4.539 & 0.62 \\
\hline $3 s-P F Y$ & & & & $3 s-P F Y$ & & & \\
\hline$\overline{\mu_{M n}}$ & 5.111 & 2.377 & 2.15 & & & & \\
\hline $45^{\circ}, 1 \mu_{\mathrm{bg}}$ & 5.111 & 2.377 & 2.15 & $45^{\circ}$ & 5.189 & 2.406 & 2.16 \\
\hline $1^{\circ}, 1 \mu_{\mathrm{bg}}$ & 5.111 & 2.377 & 2.15 & $1^{\circ}$ & 4.751 & 2.422 & 1.96 \\
\hline IPFY & & & & IPFY & & & \\
\hline$\mu_{M n}$ & 5.318 & 3.770 & 1.41 & & & & \\
\hline $45^{\circ}, 1 \mu_{\mathrm{bg}}$ & 3.209 & 2.484 & 1.29 & $45^{\circ}$ & 3.240 & 2.541 & 1.27 \\
\hline $1^{\circ}, 1 \mu_{\mathrm{bg}}$ & 4.690 & 3.493 & 1.34 & $1^{\circ}$ & 2.520 & 2.072 & 1.22 \\
\hline
\end{tabular}

\section{Reference}

(1) Henke, B. L.; Gullikson, E. M.; Davis, J. C. X-Ray Interactions: Photoabsorption, Scattering, Transmission, and Reflection at $\mathrm{E}=50-30,000 \mathrm{EV}, \mathrm{Z}=1-92$. At. Data Nucl. Data Tables 1993, 54 (2), 181-342. https://doi.org/10.1006/ADND.1993.1013. 